\title{
Blocking Peptidoglycan Recycling in Pseudomonas aeruginosa Attenuates Intrinsic Resistance to Fosfomycin
}

\author{
Marina Borisova, Jonathan Gisin, and Christoph Mayer
}

\begin{abstract}
Gram-negative bacteria recycle as much as half of their cell wall per generation. Here we show that interference with cell wall recycling in Pseudomonas aeruginosa strains results in four- to eight-fold increased susceptibility to the antibiotic fosfomycin, pushing the minimal inhibitory concentration for strains PA14 and PA01 to therapeutically appropriate values of $2-4$ and $8-16 \mathrm{mg} / \mathrm{L}$, respectively. A newly discovered metabolic pathway that connects cell wall recycling with peptidoglycan de novo biosynthesis is responsible for the high intrinsic resistance of $P$. aeruginosa to fosfomycin. The pathway comprises an anomeric cell wall amino sugar kinase (AmgK) and an uridylyl transferase (MurU), which together convert N-acetylmuramic acid (MurNAc) through MurNAc $\alpha$-1-phosphate to uridine diphosphate (UDP)-MurNAc, thereby bypassing the fosfomycinsensitive de novo synthesis of UDP-MurNAc. Thus, inhibition of peptidoglycan recycling can be applied as a new strategy for the combinatory therapy against multidrug-resistant $P$. aeruginosa strains.
\end{abstract}

\section{Introduction}

$\mathbf{T}$ HE RAPID EMERGENCE of antimicrobial drug resistance, particularly in gram-negative bacteria such as Pseudomonas aeruginosa, and the critical shortage of new antibiotics in the development against multidrug-resistant strains led to reconsider the therapeutic potential of old antibiotics. ${ }^{2,3,38}$ An example of such a well-known antibiotic that was reintroduced into clinical practice is fosfomycin. ${ }^{6,24,26,34}$ This epoxide derivative of phosphonic acid (cis-1,2-epoxypropyl phosphonic acid) was discovered in 1969, originally named phosphonomycin, and was isolated from cultures of Streptomyces species. ${ }^{11}$ Fosfomycin interferes with the first cytoplasmatic step of bacterial cell wall biosynthesis, the formation of the peptidoglycan precursor uridine diphosphate $\mathrm{N}$-acetylmuramic acid (UDP-MurNAc) ${ }^{15}$ and, thus, results in decelerated peptidoglycan synthesis, reduced growth, and eventually cell lysis. ${ }^{15,23}$ The UDP-N-acetylglucosamine (UDP-GlcNAc) enolpyruvyl transferase MurA, which catalyzes the transfer of enolpyruvate from phosphoenol pyruvate (PEP) to UDP-GlcNAc, is irreversibly inhibited by the PEPmimetic fosfomycin (Fig. 1), which covalently binds to the catalytic cysteine residue of MurA. ${ }^{16}$

Initially, fosfomycin disodium salt was applied parenterally as an alternative drug to treat patients with severe and multidrug-resistant infections. ${ }^{34}$ Later on, fosfomycin has been produced in a hydrosoluble form, fosfomycin-trometamol (also known as tromethamine or TRIS), and administrated orally for the treatment of uncomplicated urinary tract and gastrointestinal infections. ${ }^{6,32}$ Due to its long mean half-life $(5.7 \pm 2.8 \mathrm{hr})$, low toxicity, and low side effects, this drug is used alone ${ }^{25}$ or in combination with tobramycin ${ }^{37}$ to treat chronic pulmonary infections caused by multidrug-resistant $P$. aeruginosa strains in cystic fibrosis patients. Fosfomycin has been efficiently used in combination with various antimicrobial agents and in some cases, synergetic effects have been reported. ${ }^{20,28,39}$

Because of its small size (138 Da) and polarity, fosfomycin can readily cross the outer membrane of gram-negative bacteria through porins. ${ }^{10}$ In Pseudomonas sp., it is further actively transported into the cell by the glycerol-3-phosphate transporter GlpT. ${ }^{5}$ Mutations in $g l p T$ lead to decreased drug transport that result in fosfomycin resistance (reviewed in Castañeda-García et $a{ }^{4}{ }^{4}$ ). Fosfomycin resistance in Pseudomonas sp. may also be mediated by a plasmid-encoded or chromosomally encoded thiol transferase enzyme (FosA) that causes antibiotic inactivation by opening the epoxide ring of fosfomycin ${ }^{30,35,36}$ and leads to acquired drug resistance. ${ }^{27}$ Besides, $P$. aeruginosa strains generally exhibit a considerable rate of intrinsic resistance to fosfomycin. Minimal inhibitory concentrations (MICs) for fosfomycin of up to $512 \mathrm{mg} / \mathrm{L}$ in randomly selected $P$. aeruginosa strains were determined by agar dilution methods. ${ }^{7}$ According to the EUCAST criteria (URL: www.eucast .org/), $P$. aeruginosa species with a susceptibility $\geq 32 \mathrm{mg} / \mathrm{L}$ are classified as fosfomycin-resistant strains. ${ }^{19}$ 


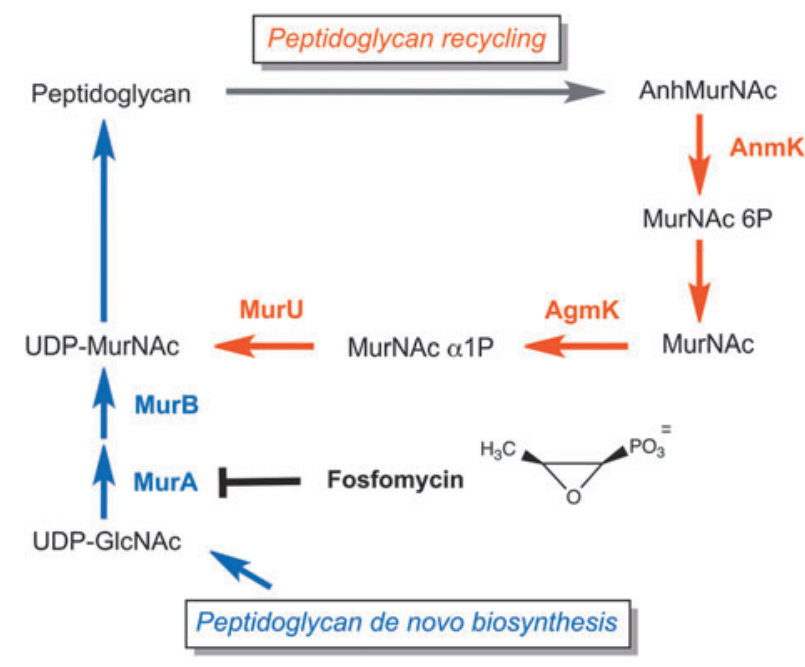

FIG. 1. Simplified scheme of the peptidoglycan recycling pathway in Pseudomonas aeruginosa. Peptidoglycan recycling (colored red) contributes to the pool of UDPMurNAc by conversion of 1,6-anhydro-MurNAc (AnhMurNAc) through the AnhMurNAc kinase (AnmK), MurNAc 6-phosphate phosphatase (not yet identified), anomeric GlcNAc/MurNAc kinase (AgmK), and MurNAc $\alpha$-1-phosphate-uridylyl transferase (MurU). Thereby, the recycling pathway bypasses de novo biosynthesis of the peptidoglycan precursor UDPMurNAc (colored blue), including the fosfomycin target MurA (chemical structure of fosfomycin).

We have recently elucidated a connection of cell wall recycling and intrinsic fosfomycin resistance in Pseudomonas putida. ${ }^{8}$ A novel cell wall salvage pathway was identified in this organism that bypasses the de novo biosynthesis of UDP-MurNAc (Fig. 1). This pathway, absent in Escherichia coli and related Enterobacteria, involves three enzymes, (1) a not-yet identified MurNAc 6-phosphate (MurNAc 6P) phosphatase, (2) an anomeric MurNAc kinase (AmgK), which forms MurNAc $\alpha$-1-phosphate (MurNAc $\alpha 1 \mathrm{P}$ ), and (3) an uridylyl transferase (MurU), which transfers uridine phosphate from UTP to the MurNAc $\alpha 1 P$, yielding UDPMurNAc. ${ }^{8}$ However, Pseudomonas sp., similar to E. coli, possess upstream of this salvage pathway enzymes for muropeptide processing, a recycling $\mathrm{N}$-acetylglucosaminidase (NagZ) that yields N-acetylglucosamine (GlcNAc) and 1,6anhydroMurNAc (AnhMurNAc) peptides, an AnhMurNAcpeptide amidase (AmpD), and an AnhMurNAc kinase (AnmK) (Fig. 1) (for more details, see Gisin et al. ${ }^{8}$ and Park and Uehara ${ }^{31}$ ). In this study, we show that the two pathogenic $P$. aeruginosa strains, PAO1 and PA14, also possess the MurNAc salvage route. Blocking this cell wall recycling pathway decreases the fosfomycin resistance of these strains at least four times, thereby pushing the MICs for the drug below the susceptibility threshold of $32 \mathrm{mg} / \mathrm{L}$. Thus, a combinatory therapy of fosfomycin along with peptidoglycan recycling inhibitors may develop into a new strategy against multidrug-resistant $P$. aeruginosa strains.

\section{Materials and Methods}

\section{Bacterial strains and culturing conditions, plasmids, and reagents}

Bacterial strains and plasmids used in this study are listed in Table 1. P. aeruginosa strain PA01 was obtained from Prof. Friedrich Götz, University of Tübingen. Strain PA14 and the complete nonredundant transposon insertion mutant library were obtained from the Broad Institute, Cambridge, MA, ${ }^{18}$ which included $\operatorname{amgK}, \operatorname{murU}$, and $a n m K$ insertion mutants (07780::MAR2xT7, 07790::MAR2xT7, and 08520::MAR2xT7 clone 06_3F4, respectively). $P$. aeruginosa and $E$. coli strains were cultured at $37^{\circ} \mathrm{C}$ in Luria-Bertani (LB) liquid or solid (plus $15 \%$ agar) medium. For antibiotic susceptibility studies, cation-adjusted Mueller-Hinton broth (Sigma-Aldrich) liquid or solid medium was applied. E-test fosfomycin strips were obtained from BioMérieux, Craponne, France. Fosfomycin disodium salt powder and Irgasan were ordered from Sigma-Aldrich. Imipenem and ceftazidime powders were from Santa Cruz Biotechnology. Phusion high-fidelity polymerase, SmaI restriction enzyme, and $E$. coli DH5 $\alpha$ cells were obtained from

Table 1. Bacterial Strains Used In This Study

\begin{tabular}{|c|c|c|}
\hline Strain & Genotype & Reference \\
\hline \multicolumn{3}{|l|}{ Pseudomonas strains } \\
\hline Pseudomonas aeruginosa PA01 & Reference strain 01 & 12 \\
\hline PA01 $\Delta 0596(\operatorname{amgK})$ & PA0596 deletion mutant of PA01 & This study \\
\hline PA01 $\Delta 0597$ (murU) & PA0597 deletion mutant of PA01 & This study \\
\hline PA01 $\Delta 0666($ anmK $)$ & PA0666 deletion mutant of PA01 & This study \\
\hline PA01 $\Delta 3005$ (nagZ) & PA3005 deletion mutant of PA01 & This study \\
\hline P. aeruginosa PA14 & Reference strain UCBPP-PA14, human isolate & \\
\hline 07780::MAR2xT7 (amgK) & $\mathrm{Gm}^{\mathrm{R}}$, PA07780 Tn insertion mutant of PA14 & 18 \\
\hline 07790::MAR2xT7 (murU) & $\mathrm{Gm}^{\mathrm{R}}, \mathrm{PA} 07790 \mathrm{Tn}$ insertion mutant of PA14 & 18 \\
\hline 08520::MAR2xT7 (06_3F4) (anmK) & $\mathrm{Gm}^{\mathrm{R}}$, PA08520 Tn insertion mutant of PA14 & 18 \\
\hline \multicolumn{3}{|l|}{ Escherichia coli strain } \\
\hline DH5 $\alpha$ & supE44 hsdR17 recA1 endA1 gyrA96 thi-1 relA1 23 & 9 \\
\hline \multicolumn{3}{|c|}{ 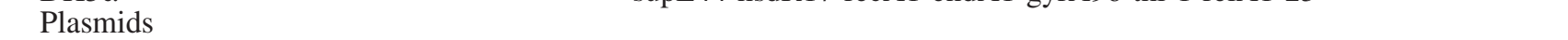 } \\
\hline pEX18Gm $\Delta 0596(\operatorname{amgK})$ & Up/Ds PA0596 in pEX18Gm, SmaI & This study \\
\hline pEX18Gm $\Delta 0597$ (murU) & Up/Ds PA0597 in pEX18Gm, SmaI & This study \\
\hline pEX18Gm $\Delta 0666($ anmK) & Up/Ds PA0666 in pEX18Gm, SmaI & This study \\
\hline pEX18Gm $\Delta 3005$ (nagZ) & Up/Ds PA3005 in pEX18Gm, SmaI & This study \\
\hline
\end{tabular}


New England Biolabs. T4 DNA ligase, genomic and plasmid DNA isolation kits, and DNA gel extraction kits were from Thermo Scientific.

\section{Construction of cell wall recycling knockout mutants}

For the generation of markerless knockout mutants in strain PA01 of genes PA0596 (MurNAc kinase), PA0597 (MurNAc 1P uridylyl transferase), PA0666 (AnhMurNAc kinase), and PA3005 (N-acetylglucosaminidase NagZ), PA01 genomic DNA was isolated according to the manufacturer's instructions. Ten nanograms of genomic DNA was used to amplify $\approx 500 \mathrm{bp}$ regions upstream and downstream of the target genes by PCR (primer pairs 1 and 2 , respectively; for the primer list, see Table 2). DNA products were purified using a polynucleotide gel extraction kit and further spliced by PCR yielding $\approx 1,000 \mathrm{bp}$ products. Subsequently, the plasmid pEX18Gm was digested with SmaI and blunt end-ligated overnight with the $\approx 1,000$ bp PCR products using T4 DNA ligase. Reactions were transformed in chemically competent $E$. coli DH5 $\alpha$ cells. ${ }^{13}$ Transformants were then selected on LB agar plates supplemented with $10 \mu \mathrm{g} / \mathrm{ml}$ gentamycin (Gm). Recombinant plasmids were isolated from single $\mathrm{DH} 5 \alpha$ transformants and the DNA sequences were checked by restriction analysis and DNA sequencing (Eurofins). The suicide plasmids (pEX18Gm $\Delta$ amgK, pEX18Gm $\Delta$ murU, and pEX18Gm $\Delta$ anmK) were further transformed in chemically competent $E$. coli helper strain $\mathrm{S} 17-1 \lambda$ pir and selected on LB agar plates with $10 \mu \mathrm{g} / \mathrm{ml} \mathrm{Gm}$. For diparental mating, $0.8 \mathrm{ml}$ of overnight cultures (incubated at $37^{\circ} \mathrm{C}$ ) of S17-1 $\lambda$ pir cells, containing the suicide plasmids, and $1.7 \mathrm{ml}$ PA01 wild-type cells, grown at $43^{\circ} \mathrm{C}$, were mixed, centrifuged, and resuspended in $200 \mu \mathrm{l}$ of final volume. The cell suspension was plated for $4 \mathrm{hr}$ at $37^{\circ} \mathrm{C}$ on membrane filters on top of the LB agar plates to allow transfer of the suicide plasmids from E. coli into Pseudomonas PA01 wildtype strains during conjugation. Filters were resuspended in $0.9 \% \mathrm{NaCl}$ and all bacteria were plated on LB supplemented with $60 \mu \mathrm{g} / \mathrm{ml} \mathrm{Gm}$ and $25 \mu \mathrm{g} / \mathrm{ml}$ Irgasan (to kill E. coli cells). Successful PA01 merodiploids were growing in the presence of antibiotic and further selected for double crossover events on LB plates without salt and 5\% sucrose. The generation of mutants was verified by the absence of growth in the presence of $\mathrm{Gm}$, colony PCR with specific primers in the flanking regions (test primers) for PA0596, PA0597, and PA0666 gene deletions (Table 2).

\section{LC-MS analysis of cell wall recycling intermediates}

PA01 wild-type and respective recycling deletion mutants, as well as PA14 wild-type and the respective recycling transposon insertion mutants were grown overnight in $50 \mathrm{ml}$ of LB medium. After $20 \mathrm{hr}$ of incubation, bacterial cultures $\left(\mathrm{OD}_{600 \mathrm{~nm}} \approx 3.2\right)$ were centrifuged $(10 \mathrm{~min}, 4,000 \mathrm{~g})$ and pellets were dissolved in $10 \mathrm{ml}$ of Tris buffer $(10 \mathrm{mM}, \mathrm{pH}$ 7.6). After two washing steps, pellets were taken up in $400 \mu \mathrm{l}$ of water and heated for $10 \mathrm{~min}$ at $95^{\circ} \mathrm{C}$ to cause cell breakage. Soluble cell extracts, containing the cell wall recycling products, were obtained by spinning down at maximum speed $(12,000 \mathrm{~g}$ for $15 \mathrm{~min})$. By adding $800 \mu \mathrm{l}$ of acetone to each $200 \mu \mathrm{l}$ of supernatant in an Eppendorf tube, protein precipitation was achieved and the remaining insoluble part was separated by centrifugation at maximum speed. The supernatant was transferred to a clean tube and dried in a speedvac at $60^{\circ} \mathrm{C}$. The dried pellet was dissolved in $160 \mu \mathrm{l}$ water before LC-MS measurement. Analysis of the accumulation of recycling products in $P$. aeruginosa mutants was conducted with a ESI-TOF mass spectrometer (MicrO-TOF II, Bruker) that was connected to the Ultimate

Table 2. Collection of Nucleotide Primers

\begin{tabular}{|c|c|c|}
\hline Primers & Primer sequence $5^{\prime}-3^{\prime}$ & Application \\
\hline $0596 \mathrm{Fw}$ pair1 & GGCGGTCAGTTTTTCCCGAG & $\operatorname{amg} K$ deletion \\
\hline 0596 Rev pair1 & TCGGCTCCCTGCGGTTCTCCACGGCCCTAGC & \\
\hline 0596 Fw pair2 & CCGTGGAGAACCGCAGGGAGCCGAGGCATGAA & \\
\hline 0596 Rev pair2 & CAGTACGGCGATCCCGCTG & \\
\hline $0596 \mathrm{Fw}$ test & TTGGCGGATACGTAGGTCGG & \\
\hline 0596 Rev test & GCCAGCAATCGCTCGACTTC & \\
\hline 0597 Fw pair1 & GCCCGCTGGAAACGGATCTG & murU deletion \\
\hline 0597 Rev pair1 & AGCGGGCCAGAGCATGCCTCGGCTCCCTGC & \\
\hline $0597 \mathrm{Fw}$ pair2 & AGCCGAGGCATGCTCTGGCCCGCTACGCTGATCG & \\
\hline 0597 Rev pair2 & GTTCGCTCCGCCGACCAAC & \\
\hline $0597 \mathrm{Fw}$ test & CCTTCCAGAAGGTCGATGTC & \\
\hline 0597 Rev test & GCCAGTTTGTCCGGATGATG & \\
\hline 0666 Fw pair1 & GTGGATTTCGCCCGTATCAG & anm $K$ deletion \\
\hline 0666 Rev pair1 & GGACCGAGCGCAATCAGCGCTGCTTGTTCAGGG & \\
\hline 0666 Fw pair2 & AGCAGCGCTGATTGCGCTCGGTCCCAGAACTC & \\
\hline 0666 Rev pair2 & CTGGGACAAGAGGATAATGG & \\
\hline 0666 Fw test & TCAACCGTGGCAAGACCTAC & \\
\hline 0666 Rev test & CATGTGATCATCGACGGTCAG & \\
\hline $3005 \mathrm{Fw}$ pair1 & CTACATGCTGCTGGTCAAC & nagZ deletion \\
\hline 3005 Rev pair1 & ATCAGTTGCGCACCGATGTCGAGCATCAGAG & \\
\hline $3005 \mathrm{Fw}$ pair2 & GCTCGACATCGGTGCGCAACTGATTGATTGAGGG & \\
\hline 3005 Rev pair2 & TAGACCCCATGGCTGCTGTG & \\
\hline
\end{tabular}

Overlapping regions of primer pairs are shown in bold. 
3000 HPLC system (Dionex), as previously described. ${ }^{8}$ For high-performance liquid chromatography (HPLC) separation, a Gemini C18 column (Phenomenex, $150 \times 4.6 \mathrm{~mm}, 110 \AA, 5 \mu \mathrm{M}$ ) was used. Samples ( $5 \mu \mathrm{l}$ injection volume) were applied to the HPLC column at a flow rate of $0.2 \mathrm{ml} / \mathrm{min}$ and using a $45-\mathrm{min}$ program (for $5 \mathrm{~min}, 100 \%$ buffer A: $0.1 \%$ formic acid with $0.05 \%$ ammonium formate, then $30 \mathrm{~min}$ of a linear gradient to $40 \%$ buffer B with $100 \%$ acetonitrile, 5 min of $40 \%$ buffer B, and $5 \mathrm{~min}$ of re-equilibration step). Data are presented as differential ion chromatograms $(\Delta \mathrm{TIC})$. These were obtained by subtracting the total ion chromatogram (TIC) of a mutant cell extract minus the TIC of the respective wildtype extract, calculated by automatically subtracting TICs using the program called "Metabolite Detect" (Bruker).

\section{Determination of the MICs}

MICs for $P$. aeruginosa strains PA01 and PA14 were determined by using the broth microdilution method, according to the Clinical and Laboratory Standards Institute (CLSI) recommendations or by applying E-test fosfomycin strips following the manufacturer's instructions. Briefly, the broth microdilution method was performed in U-form 96well plates (VWR) using the Mueller-Hinton Broth (cationadjusted) medium in a total volume of $100 \mu \mathrm{l}$ and a shaking incubator at $35^{\circ} \mathrm{C}, 160 \mathrm{rpm}$ for $20 \mathrm{hr}$ in a humidified chamber. Bacteria were harvested at an optical density $\left(\mathrm{OD}_{600 \mathrm{~nm}}\right)$ of 0.3 and diluted to a starter culture of $1 \times 10^{4}$ cells. Appropriate dilution series of the antibiotic fosfomycin (FOS, 0.5-256 mg/ml), imipenem (IMP, 0.0625-8 mg/L), and ceftazidime (CAZ, $0.0625-8 \mathrm{mg} / \mathrm{L}$ ) were previously diluted in the broth medium. The absence of growth at the lowest used antibiotic concentration was determined as the MIC value.
For E-tests, fosfomycin strips with a dilution range between 0.064 and 1,024 $\mathrm{mg} / \mathrm{ml}$ and Mueller-Hinton broth (with $\mathrm{Ca}$ and $\mathrm{Mg}$ ions) agar plates $(17.5 \mathrm{ml})$ were used. Pseudomonas overnight cultures were adjusted with $0.9 \%$ $\mathrm{NaCl}$ to $0.5 \mathrm{McFarland}$ standard and bacteria were transferred with a sterile loop to the plates. Results were documented after incubation at $37^{\circ} \mathrm{C}$ for $20 \mathrm{hr}$.

\section{Results and Discussion}

\section{Peptidoglycan recycling in $\mathrm{P}$. aeruginosa involves a direct shortcut pathway to UDP-MurNAC}

We first aimed to investigate whether peptidoglycan recycling in $P$. aeruginosa PA01 and PA14 involves orthologs of the newly discovered recycling genes amg $K$ and mur $U$ of $P$. putida. If so, we expected that mutants for these genes will accumulate specific recycling intermediates, as was previously shown for $P$. putida ${ }^{8}$ Therefore, we generated markerless deletion mutants in PA01 of genes PA0596 ( $\Delta a m g K)$ and PA0597 ( $\Delta m u r U)$, as well as deletion mutants of known recycling genes, PA3005 $(\Delta$ nagZ) and PA0666 $(\Delta a n m K)$. In addition, transposon insertion mutants were obtained from the PA14 mutant collection $^{18}$ of the respective genes of $P$. aeruginosa strain PA14 (except for PA14 nagZ mutant, which is not available in this collection). We demonstrated by LCMS that MurNAc accumulates in the cell extract of amgK mutants (Fig. 2A) and MurNAc 1P accumulates in the cell extract of murU mutants (Fig. 2B), similar to observed accumulation products in $P$. putida orthologous mutants. ${ }^{8}$ Moreover, AnhydroMurNAc was shown to accumulate in the anmK mutant strains (Fig. 2C). Thus, these results indicate that AmgK and MurU in
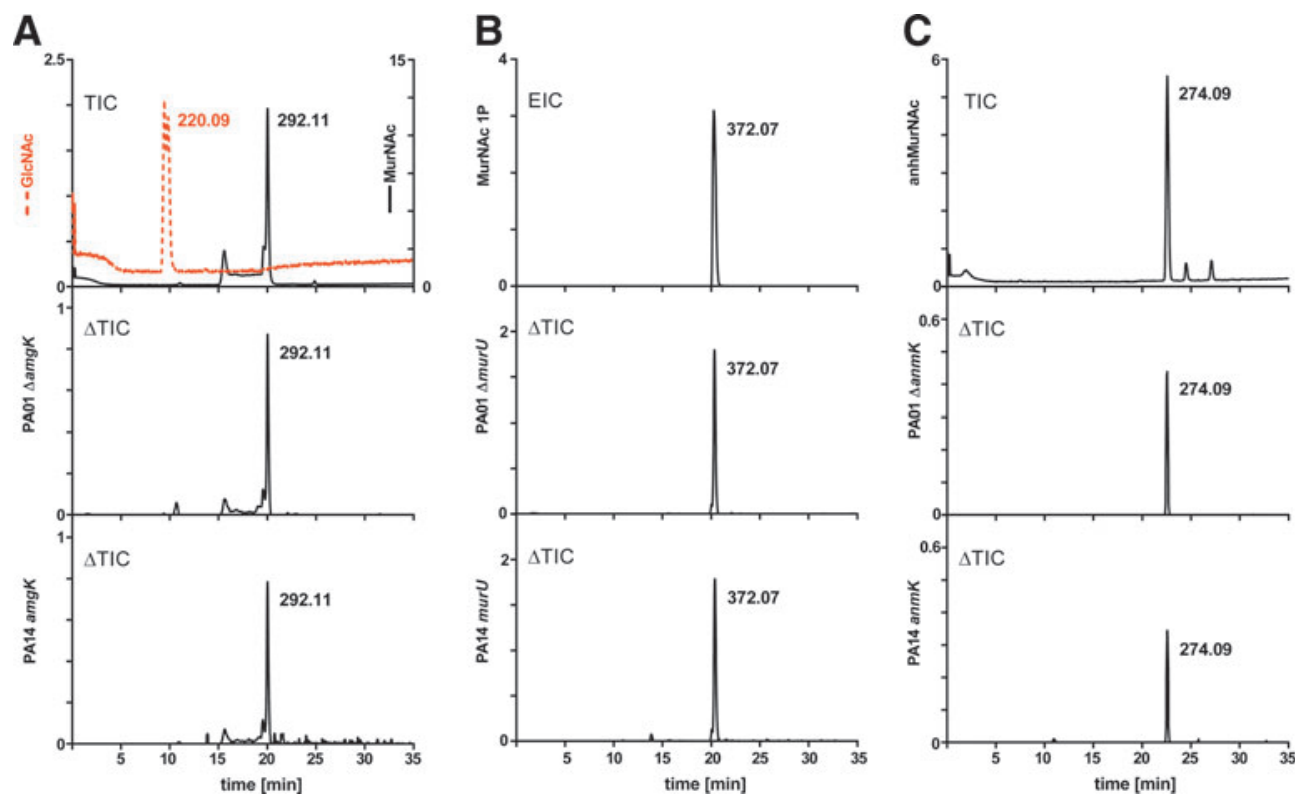

FIG. 2. Identification of cell wall recycling products by LC-MS analysis. Total ion chromatograms (TICs) were obtained in the negative ion mode; the y axes represent ion intensity (counts per second). (A) Overlay of GlcNAc (m/z=220.09; colored red) and MurNAc $(\mathrm{m} / \mathrm{z}=292.11), 5 \mathrm{mM}$ each, and differential ion chromatograms $(\Delta \mathrm{TICs})$ obtained by subtracting TIC of mutant (PA01 $\triangle a m g K$ or PA14 $a m g K:: T n$ ) from the respective wild type. (B) Extracted ion chromatogram (EIC) for MurNAc 1P $(\mathrm{m} / \mathrm{z}=372.07)$ and PA01 $\Delta$ murU and PA14 murU::Tn $\Delta$ TICs. (C) TIC of AnhMurNAc $(\mathrm{m} / \mathrm{z}=274.09), 1 \mathrm{mM}$, and PA01 $\triangle a n m K$ and PA14 anmK::Tn $\Delta$ TICs. 

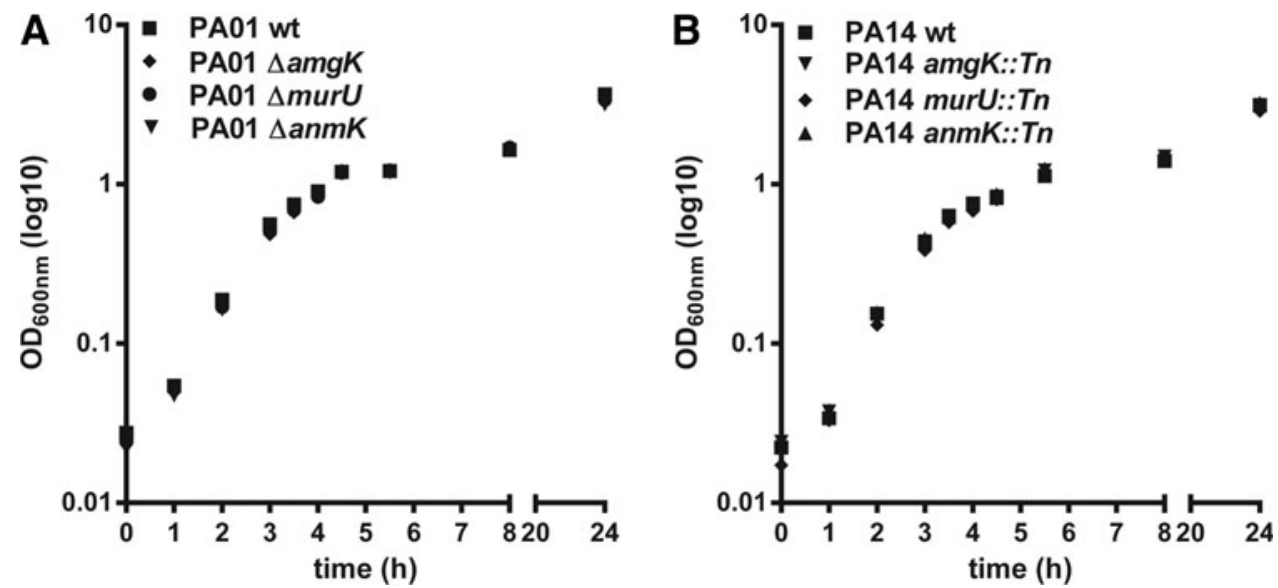

FIG. 3. Growth fitness comparison of $P$. aeruginosa strains in the LB medium. Optical densities $\left(\mathrm{OD}_{600 \mathrm{~nm}}\right)$ are presented as base $10 \operatorname{logarithms}(\log 10)$. (A) PA01 wild-type and respective deletion mutants $(\Delta a m g K, \Delta m u r U$, and $\Delta a n m K)$. (B) PA14 wild-type and respective transposon (Tn) insertion mutants (amgK::Tn, murU::Tn, and anmK::Tn). Results are shown as a mean $\pm \mathrm{SD}$ of two independent experiments, each done in duplicate.

$P$. aeruginosa constitute a shortcut recycling pathway, contributing to the UDP-MurNAc pool.

\section{Blocking peptidoglycan recycling in $\mathrm{P}$. aeruginosa does not affect growth}

Growth of Pseudomonas wild-type as well as $\operatorname{amgK}$, $m u r U$, and anmK mutants was compared using the LB medium. Identical growth rates were observed for wild-type (PA01 and PA14) and respective recycling mutant strains (Fig. 3A, B), indicating that blocking cell wall recycling does not affect general fitness in a rich medium. Previous studies comparing wild-type and recycling mutants in $E$. coli and $P$. putida also revealed no difference in growth rates. ${ }^{8,31}$ Since the peptidoglycan layer of $E$. coli, and presumably of $P$. aeruginosa as well, accounts for only about $2 \%$ of the cell mass, ${ }^{22}$ Park and Uehara suggested that cell wall recycling is not essential for cell viability and growth. ${ }^{31}$ Still, as shown by LC-MS results (Fig. 2), the recycling pathway through AnmK, AmgK, and MurU channels cell wall recycling fragments directly into the UDP-MurNAc pool and, thus, bypasses the MurA-catalyzed enzymatic reaction that is the target of fosfomycin (cf. Fig. 1). Cell wall recycling therefore contributes to the intrinsic fosfomycin resistance in $P$. aeruginosa.

\section{Pseudomonas cell wall recycling mutants are more susceptible to fosfomycin compared with wild type}

The contribution of cell wall recycling to the UDPMurNAc pool, and thus, the intrinsic resistance to fosfomycin, was shown by comparison of the MIC values for Pseudomonas wild-type and mutant strains (Table 3). By using the broth microdilution method, we observed that the MIC value for fosfomycin of $32 \mathrm{mg} / \mathrm{L}$ for the PA01 wild type was reduced to $8 \mathrm{mg} / \mathrm{L}$ in $\Delta a m g K, \Delta m u r U, \Delta a n m K$, and $\triangle$ nagZ deletion mutants. A fourfold increase in fosfomycin susceptibility in the absence of the indicated PA01 recycling genes was also seen by applying E-test strips; the MICs dropped from $64 \mathrm{mg} / \mathrm{L}$ in PA01 wild-type to $16 \mathrm{mg} / \mathrm{L}$ in the recycling mutants.
Overall, fosfomycin susceptibility of strain PA14 was two to three times higher compared with PA01. Still, fosfomycin sensitivity was similarly increased in the PA14 cell wall recycling mutants. Broth microdilution tests showed a MIC value of $16 \mathrm{mg} / \mathrm{L}$ for the PA14 wild-type strain, and eightfold decreased MICs (2 mg/L) in the PA14 anmK, $\operatorname{amgK}$, and murU transposon insertion mutants. E-test strips confirmed these results. With this test, a MIC value of $24 \mathrm{mg} / \mathrm{L}$ for fosfomycin was determined in the PA14 wild type, and a value of $4 \mathrm{mg} / \mathrm{L}$ in the absence of recycling genes (a sixfold increase in fosfomycin sensitivity).

We wondered whether the fosfomycin sensitivity of cell wall recycling mutants is a specific or a general stress response. Therefore, we tested the susceptibility of PA01 wild type and $\Delta a m g K, \Delta m u r U, \Delta a n m K$, and $\Delta n a g Z$ mutants toward the $\beta$-lactam antibiotics, ceftazidime (a 3rd generation

Table 3. Comparison of Fosfomycin and $\beta$-Lactam Resistances IN $P$. AERUGINOSA WILD-TyPE and Recycling Mutant Strains

\begin{tabular}{|c|c|c|c|c|}
\hline \multirow[b]{2}{*}{ Strain } & \multicolumn{4}{|c|}{$M I C(m g / L)$} \\
\hline & $F O S^{\mathrm{a}}$ & $F O S^{\mathrm{b}}$ & $C A Z^{\mathrm{a}}$ & $I M P^{\mathrm{a}}$ \\
\hline PA01 wild type & 32 & 64 & 1 & 1 \\
\hline PA01 $\Delta a m g K$ & 8 & 16 & 1 & 1 \\
\hline PA01 $\Delta m u r U$ & 8 & 16 & 1 & 1 \\
\hline PA01 $\triangle a n m K$ & 8 & 16 & 1 & 1 \\
\hline PA01 $\Delta n a g Z$ & 8 & 16 & 1 & 1 \\
\hline PA14 wild type & 16 & 24 & nd & nd \\
\hline PA14 amgK $:: T n$ & 2 & 4 & nd & nd \\
\hline PA14 murU::Tn & 2 & 4 & nd & nd \\
\hline PA14 anmK $:: T n$ & 2 & 4 & nd & nd \\
\hline
\end{tabular}

Sensitivity to fosfomycin (FOS) and the $\beta$-lactams ceftazidime (CAZ) and imipenem (IMP) was assayed using either the broth microdilution method (FOS, ${ }^{\mathrm{a}} \mathrm{CAZ},{ }^{\mathrm{a}}$ and $\mathrm{IMP}^{\mathrm{a}}$ ) or E-test strips for fosfomycin $\left(\mathrm{Fos}^{\mathrm{b}}\right)$.

${ }^{a}$ Represents results of two independent experiments, each done in quadruplets.

${ }^{\mathrm{b}}$ Represents the mean of three independent experiments.

nd, not defined. 
cephalosporin) and imipenem (a carbapenem), both having inhibitory effects on $P$. aeruginosa. ${ }^{21}$ We obtained identical MIC values $(1 \mathrm{mg} / \mathrm{L})$ for the two antibiotics in wild-type and mutant strains (Table 3). This indicates that blocking cell wall recycling does not generally affect susceptibility to antimicrobial drugs.

\section{Application of fosfomycin in combinatory therapy}

Spontaneous mutations in the cell wall recycling pathway are a common cause of acquired resistance to $\beta$-lactam antibiotics in $P$. aeruginosa clinical isolates. ${ }^{40}$ A specific cell wall recycling product, AnhMurNAc-pentapeptide, was shown to accumulate in these isolates, acting as an inducer of the chromosomally encoded AmpC $\beta$-lactamase. ${ }^{14,21}$ Hyperproduction of AmpC was attenuated by blocking cell wall recycling at the level of NagZ, and thus, $\beta$-lactam susceptibility was restored. ${ }^{14,40}$ We showed that the PA01 $\Delta$ nagZ mutant, similar to the other recycling mutants, is four times more susceptible to fosfomycin compared with wild type (Table 3 ). However, the sensitivity toward the $\beta$ lactams ceftazidime and imipenem was not affected in the $\triangle$ nagZ mutant (Table 3), in agreement with previous reports. ${ }^{21,40}$ Nevertheless, in multidrug-resistant clinical isolates, inhibition of cell wall recycling may be applied in a combinatory therapy with fosfomycin to reduce its intrinsic resistance, and simultaneously, the acquired resistance to certain $\beta$-lactams.

\section{Conclusions}

$P$. aeruginosa is a problematic opportunistic pathogen, particularly due to its high intrinsic resistance to many antibiotics. In addition to the mechanisms that traditionally have been attributed to intrinsic resistance of $P$. aeruginosa, such as low permeability, efficient detoxification by efflux pumps, and antibiotic inactivation enzymes, recent studies indicate that intrinsic antibiotic resistance should be considered as an emergent systemic property rather than a specific adaptation to the presence of antibiotics. ${ }^{1,17,29}$ In this vein, the novel cell wall recycling route in Pseudomonas sp. ${ }^{8}$ provides a rationale for intrinsic resistance to fosfomycin, that is, the consequence of a unique metabolic pathway of cell wall sugar recovery in this organisms. We suggest compounds that interfere with cell wall recycling can be used to increase fosfomycin sensitivity and may be applied in combination to treat multidrug-resistant $P$. aeruginosa.

\section{Acknowledgments}

This work was supported by grants to CM of the BadenWürttemberg Stiftung (P-BWS-Glyko11), and the German Research Foundation (DFG grant MA2436/4 and SFB766/ A15). JG gratefully acknowledges a "Dr. Marietta-LutzeStipend," of the company Dr. Kade AG (Konstanz and Berlin). Special thanks go to Alexander Schneider (Univ. of Tübingen) for valuable LC-MS advice, to Prof. Herbert P. Schweizer for pEX18Gm plasmid, and to Kai Thormann for S17-1 $\lambda$ pir strain. The authors also thank Prof. Friedrich Götz (Univ. of Tübingen) for the PA01 strain and for the help from his technical team (Daniel Kühner and Regine Stemmler).

\section{Disclosure Statement}

No competing financial interests exist.

\section{References}

1. Alvarez-Ortega, C., I. Wiegand, J. Olivares, R.E. Hancock, and J.L. Martinez. 2010. Genetic determinants involved in the susceptibility of Pseudomonas aeruginosa to $\beta$-lactam antibiotics. Antimicrob. Agents Chemother. 54: 4159-4167.

2. Bassetti, M., F. Ginocchio, M. Mikulska, L. Taramasso, and D.R. Giacobbe. 2011. Will new antimicrobials overcome resistance among Gram-negatives? Expert. Rev. Anti. Infect. Ther. 9:909-922.

3. Bergen, P.J., C.B. Landersdorfer, H.J. Lee, J. Li, and R.L. Nation. 2012. 'Old' antibiotics for emerging multidrug-resistant bacteria. Curr. Opin. Infect. Dis. 25:626633.

4. Castañeda-García, A., J. Blázquez, and A. RodríguezRojas. 2013. Molecular mechanisms and clinical impact of acquired and intrinsic fosfomycin resistance. Antibiotics. 2:217-236.

5. Castaneda-Garcia, A., A. Rodriguez-Rojas, J.R. Guelfo, and J. Blazquez. 2009. The glycerol-3-phosphate permease GlpT is the only fosfomycin transporter in Pseudomonas aeruginosa. J. Bacteriol. 191:6968-6974.

6. Falagas, M.E., K.P. Giannopoulou, G.N. Kokolakis, and P.I. Rafailidis. 2008. Fosfomycin: use beyond urinary tract and gastrointestinal infections. Clin. Infect. Dis. 46:10691077.

7. Falagas, M.E., M.D. Kanellopoulou, D.E. Karageorgopoulos, G. Dimopoulos, P.I. Rafailidis, N.D. Skarmoutsou, and E.A. Papafrangas. 2008. Antimicrobial susceptibility of multidrug-resistant Gram negative bacteria to fosfomycin. Eur. J. Clin. Microbiol. Infect. Dis. 27:439-443.

8. Gisin, J., A. Schneider, B. Nagele, M. Borisova, and C. Mayer. 2013. A cell wall recycling shortcut that bypasses peptidoglycan de novo biosynthesis. Nat. Chem. Biol. 9: 491-493.

9. Hanahan, D. 1983. Studies on transformation of Escherichia coli with plasmids. J. Mol. Biol. 166:557-580.

10. Hancock, R.E., R. Siehnel, and N. Martin. 1990. Outer membrane proteins of Pseudomonas. Mol. Microbiol. 4: 1069-1075.

11. Hendlin, D., E.O. Stapley, M. Jackson, H. Wallick, A.K. Miller, F.J. Wolf, T.W. Miller, L. Chaiet, F.M. Kahan, E.L. Foltz, et al. 1969. Phosphonomycin, a new antibiotic produced by strains of streptomyces. Science. 166:122123.

12. Holloway, B.W. 1955. Genetic recombination in Pseudomonas aeruginosa. J. Gen. Microbiol. 13:572-581.

13. Inoue, H., H. Nojima, and H. Okayama. 1990. High efficiency transformation of Escherichia coli with plasmids. Gene. 96:23-28.

14. Jacobs, C., J.M. Frere, and S. Normark. 1997. Cytosolic intermediates for cell wall biosynthesis and degradation control inducible $\beta$-lactam resistance in gram-negative bacteria. Cell. 88:823-832.

15. Kahan, F.M., J.S. Kahan, P.J. Cassidy, and H. Kropp. 1974. The mechanism of action of fosfomycin (phosphonomycin). Ann. N. Y. Acad. Sci. 235:364-386.

16. Kim, D.H., W.J. Lees, K.E. Kempsell, W.S. Lane, K. Duncan, and C.T. Walsh. 1996. Characterization of a 
Cys115 to Asp substitution in the Escherichia coli cell wall biosynthetic enzyme UDP-GlcNAc enolpyruvyl transferase (MurA) that confers resistance to inactivation by the antibiotic fosfomycin. Biochemistry. 35:4923-4928.

17. Krahn, T., C. Gilmour, J. Tilak, S. Fraud, N. Kerr, C.H. Lau, and K. Poole. 2012. Determinants of intrinsic aminoglycoside resistance in Pseudomonas aeruginosa. Antimicrob. Agents Chemother. 56:5591-5602.

18. Liberati, N.T., J.M. Urbach, S. Miyata, D.G. Lee, E. Drenkard, G. Wu, J. Villanueva, T. Wei, and F.M. Ausubel. 2006. An ordered, nonredundant library of Pseudomonas aeruginosa strain PA14 transposon insertion mutants. Proc. Natl. Acad. Sci. U. S. A. 103:2833-2838.

19. Lu, C.L., C.Y. Liu, Y.T. Huang, C.H. Liao, L.J. Teng, J.D. Turnidge, and P.R. Hsueh. 2011. Antimicrobial susceptibilities of commonly encountered bacterial isolates to fosfomycin determined by agar dilution and disk diffusion methods. Antimicrob. Agents Chemother. 55:4295-4301.

20. MacLeod, D.L., L.M. Barker, J.L. Sutherland, S.C. Moss, J.L. Gurgel, T.F. Kenney, J.L. Burns, and W.R. Baker. 2009. Antibacterial activities of a fosfomycin/tobramycin combination: a novel inhaled antibiotic for bronchiectasis. J. Antimicrob. Chemother. 64:829-836.

21. Mark, B.L., D.J. Vocadlo, and A. Oliver. 2011. Providing $\beta$-lactams a helping hand: targeting the AmpC $\beta$-lactamase induction pathway. Future Microbiol. 6:1415-1427.

22. Matias, V.R., A. Al-Amoudi, J. Dubochet, and T.J. Beveridge. 2003. Cryo-transmission electron microscopy of frozen-hydrated sections of Escherichia coli and Pseudomonas aeruginosa. J. Bacteriol. 185:6112-6118.

23. Mengin-Lecreulx, D., and J. van Heijenoort. 1990. Correlation between the effects of fosfomycin and chloramphenicol on Escherichia coli. FEMS Microbiol. Lett. 54: 129-133.

24. Michalopoulos, A.S., I.G. Livaditis, and V. Gougoutas. 2011. The revival of fosfomycin. Int. J. Infect. Dis. 15: e732-e739.

25. Mirakhur, A., M.J. Gallagher, M.J. Ledson, C.A. Hart, and M.J. Walshaw. 2003. Fosfomycin therapy for multiresistant Pseudomonas aeruginosa in cystic fibrosis. J. Cyst. Fibros. 2:19-24.

26. Neuner, E.A., J. Sekeres, G.S. Hall, and D. van Duin. 2012. Experience with fosfomycin for treatment of urinary tract infections due to multidrug-resistant organisms. Antimicrob. Agents Chemother. 56:5744-5748.

27. Nikolaidis, I., S. Favini-Stabile, and A. Dessen. 2014. Resistance to antibiotics targeted to the bacterial cell wall. Protein Sci. 23:243-259.

28. Okazaki, M., K. Suzuki, N. Asano, K. Araki, N. Shukuya, T. Egami, Y. Higurashi, K. Morita, H. Uchimura, and T. Watanabe. 2002. Effectiveness of fosfomycin combined with other antimicrobial agents against multidrug-resistant Pseudomonas aeruginosa isolates using the efficacy time index assay. J. Infect. Chemother. 8:37-42.

29. Olivares, J., A. Bernardini, G. Garcia-Leon, F. Corona, M.B. Sanchez, and J.L. Martinez. 2013. The intrinsic resistome of bacterial pathogens. Front Microbiol. 4:103.
30. Pakhomova, S., C.L. Rife, R.N. Armstrong, and M.E. Newcomer. 2004. Structure of fosfomycin resistance protein FosA from transposon Tn2921. Protein Sci. 13:12601265.

31. Park, J.T., and T. Uehara. 2008. How bacteria consume their own exoskeletons (turnover and recycling of cell wall peptidoglycan). Microbiol. Mol. Biol. Rev. 72:211-227.

32. Patel, S.S., J.A. Balfour, and H.M. Bryson. 1997. Fosfomycin tromethamine. A review of its antibacterial activity, pharmacokinetic properties and therapeutic efficacy as a single-dose oral treatment for acute uncomplicated lower urinary tract infections. Drugs. 53:637-656.

33. Rahme, L.G., E.J. Stevens, S.F. Wolfort, J. Shao, R.G. Tompkins, and F.M. Ausubel. 1995. Common virulence factors for bacterial pathogenicity in plants and animals. Science. 268:1899-1902.

34. Raz, R. 2012. Fosfomycin: an old-new antibiotic. Clin. Microbiol. Infect. 18:4-7.

35. Rife, C.L., R.E. Pharris, M.E. Newcomer, and R.N. Armstrong. 2002. Crystal structure of a genomically encoded fosfomycin resistance protein (FosA) at 1.19 A resolution by MAD phasing off the L-III edge of $\mathrm{Tl}(+)$. J. Am. Chem. Soc. 124:11001-11003.

36. Rigsby, R.E., C.L. Rife, K.L. Fillgrove, M.E. Newcomer, and R.N. Armstrong. 2004. Phosphonoformate: a minimal transition state analogue inhibitor of the fosfomycin resistance protein, FosA. Biochemistry. 43:13666-13673.

37. Trapnell, B.C., S.A. Mc Colley, D.G. Kissner, M.W. Rolfe, J.M. Rosen, M. McKevitt, L. Moorehead, A.B. Montgomery, and D.E. Geller. 2012. Fosfomycin/tobramycin for inhalation in patients with cystic fibrosis with pseudomonas airway infection. Am. J. Respir. Crit. Care Med. 185:171-178.

38. Velkov, T., K. Roberts, R. Nation, P. Thompson, and J. Li. 2013. Pharmacology of polymyxins: new insights into an 'old' class of antibiotics. Future Microbiol. 8:711-724.

39. Yamada, S., Y. Hyo, S. Ohmori, and M. Ohuchi. 2007. Role of ciprofloxacin in its synergistic effect with fosfomycin on drug-resistant strains of Pseudomonas aeruginosa. Chemotherapy. 53:202-209.

40. Zamorano, L., T.M. Reeve, L. Deng, C. Juan, B. Moya, G. Cabot, D.J. Vocadlo, B.L. Mark, and A. Oliver. 2010. NagZ inactivation prevents and reverts beta-lactam resistance, driven by AmpD and PBP 4 mutations, in Pseudomonas aeruginosa. Antimicrob. Agents Chemother. 54: 3557-3563.

Address correspondence to: Christoph Mayer, PhD Department of Biology Interfaculty Institute of Microbiology and Infection Medicine Tübingen University of Tübingen Tübingen 72076

Germany

E-mail: christoph.mayer@uni-tuebingen.de 